\title{
Molecular Doping of Electrochemically Prepared Triazine-Based Carbon Nitride by 2,4,6-Triaminopyrimidine for Improved Photocatalytic Properties
}

\author{
Leonard Heymann, ${ }^{\dagger} \odot$ Sophia C. Bittinger, ${ }^{\dagger}$ and Christian Klinke ${ }^{*},+\neq \odot$ \\ ${ }^{\dagger}$ Institute of Physical Chemistry, University of Hamburg, Martin-Luther-King-Platz 6, 20146 Hamburg, Germany \\ "Chemistry Department, Swansea University, Singleton Park, SA2 8PP Swansea, U.K.
}

Supporting Information

ABSTRACT: Copolymerization of melamine with 2,4,6triaminopyrimidine (TAP) in an electrochemically induced polymerization process leads to the formation of molecular doped poly(triazine imide) (PTI). The polymerization is based on the electrolysis of water and evolving radicals during this process. The incorporation of TAP is shown by techniques such as elemental analysis, Fourier transform infrared and NMR spectroscopies, and powder X-ray diffraction, and it is shown that the carbon content can be tuned by the variation of the molar ratio of the two precursors. This incorporation of TAP directly influences the electronic

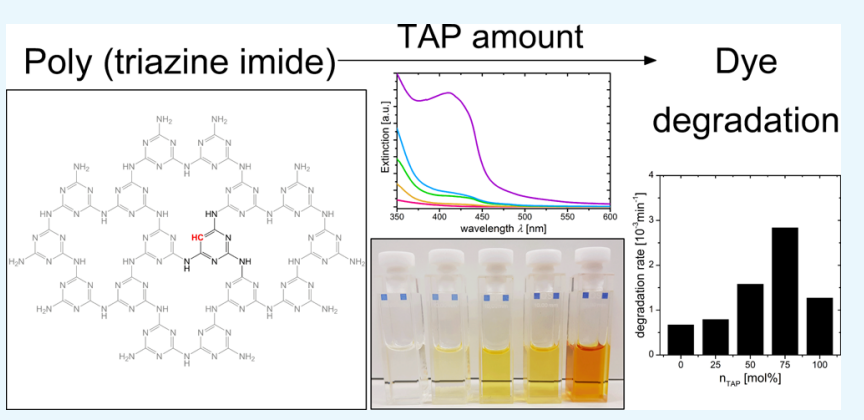
structure of PTI and as a result, a red shift can be observed in UV-vis spectroscopy. The smaller band gap and the increased absorption in the visible range lead to improved photocatalytic properties. In dye degradation experiments, it was possible to observe an increase of the rate of the degradation of methylene blue by a factor of 4 in comparison to undoped PTI or 7 if compared to melon.

\section{INTRODUCTION}

Carbon nitrides as nontoxic and metal-free photocatalysts have been of particular interest in the scientific community since the discovery of photocatalytic water splitting by Wang et al. in 2009. ${ }^{1}$ The different degrees of polymerization and their either triazine or heptazine-based structure can be obtained by different synthesis procedures. These synthesis procedures lead to carbon nitride materials such as melon (often referred as g$\left.\mathrm{C}_{3} \mathrm{~N}_{4}\right),{ }^{2-5}$ poly(triazine imide) (PTI), ${ }^{6-9}$ poly(heptazine imide), ${ }^{10-12}$ and triazine-based graphitic carbon nitride. ${ }^{13-15}$

Different paths were followed to improve the photocatalytic properties of these carbon nitrides. One example is to increase the specific surface and to modify the morphology to increase the number of accessible active sites. This can be done by exfoliation or by soft or hard template methods, which often results in improved photocatalytic properties. ${ }^{16-18}$ Doping can be divided into molecular and elemental doping. Molecular doping describes the incorporation of different molecules, for example barbituric acid and 2,4,6-triaminopyrimidine (TAP). ${ }^{19-23}$ Another promising molecule for molecular doping is 2-aminobenzonitrile, which was used by Zhang et al. to dope $g-\mathrm{C}_{3} \mathrm{~N}_{4}$. This doped carbon nitride has an increased absorption in the visible range and improved photocatalytic properties in hydrogen evolution experiments. ${ }^{24}$ Elemental doping is achieved by the substitution of single atoms in the triazine or heptazine building unit by elements such as $\mathrm{O},{ }^{25}$ $\mathrm{C},{ }^{26} \mathrm{P},{ }^{27} \mathrm{~S},{ }^{28} \mathrm{~B},{ }^{29,30} \mathrm{I},{ }^{31,32}$ and $\mathrm{F} .{ }^{33,34}$ Also, the introduction of metals such as $\mathrm{Fe}$ into carbon nitrides has been reported by Wang et al., which is described as another approach to effectively tune the photocatalytic properties of carbon nitrides. $^{35}$

In this work, we focus on molecular doping of PTI and discuss a facile variation of a novel synthesis that has been recently reported by the authors. ${ }^{36}$ This approach is conducted in an aqueous solution of melamine, in which between two platinum electrodes a voltage is applied, which exceeds the potential of the electrolysis of water. During the process of electrolysis, radical species such as hydroxyl or superoxide radicals are formed. These radicals react with the melamine precursor to form melamine radicals which polymerize. ${ }^{37}$ In further experiments, it was shown that hydroxyl radicals are expected to be the main species in this reaction. ${ }^{36}$ Through step-wise substituting melamine by TAP in this approach, it was possible to tune the optical properties of PTI, leading to a significant improvement of the photocatalytic properties. For the degradation of methylene blue (MB), a significant increase of the degradation rate could be observed with an increasing amount of TAP.

Received: October 5, 2018

Accepted: November 28, 2018

Published: December 11, 2018 


\section{RESULTS AND DISCUSSION}

In order to achieve the molecular doping of PTI as it is shown in Figure 1, melamine was substituted by TAP. Therefore,

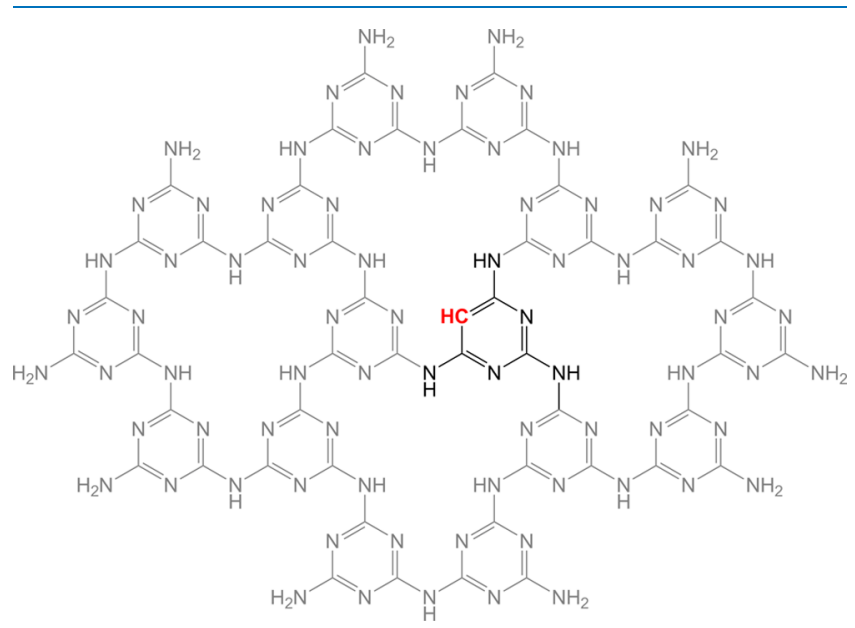

Figure 1. Structure of PTI is illustrated in gray. An s-triazine unit, which has been substituted by a pyrimidine unit, is shown in black. The exchange of nitrogen by carbon is marked red.

solutions were prepared with $0,25,50,75$, and $100 \mathrm{~mol} \%$ of TAP. The total molar amount and the sodium hydroxide concentration of melamine and TAP were constant. The reactions were conducted in a vessel shown in the photograph in Figure SI1. The reactions were followed by UV-vis spectroscopy for the evaluation of the reaction times. In accordance to the Lambert-Beer law, the reactions were considered to be completed when there were no further changes in the absorption spectra, thus depicting a constant concentration of the product. These spectra in Figure SI2 show that the reaction time increases with the amount of TAP used in synthesis. After precipitation using hydrochloric acid and a washing procedure, the product was freeze-dried and the incorporation was demonstrated by applying different characterization methods.

The powder X-ray diffraction (XRD) pattern is shown in Figure 2. It consists of two reflexes. The reflex at $27.75^{\circ}$ is related to the (002) stacking of conjugated aromatic systems. ${ }^{1}$ The calculated interlayer distance is found to be $3.22 \AA$. With an increasing amount of TAP substituting melamine in the synthesis, the position is at a constant angle with an exception of the completely substituted sample, which shows a slight shift to smaller angles $\left(27.59^{\circ}\right)$. This behavior has been observed for other carbon nitrides and is explained by the substitution of a nitrogen atom, resulting in reduced stability and structural regularity. ${ }^{19,22}$ The increase of the full width half maximum (fwhm) indicates that the stacking of the single-layer PTI is decreased and smaller crystal domains are formed. Using the Scherrer equation, sizes of $19.1 \mathrm{~nm}$ are found for PTI synthesized using melamine, whereas the complete substitution leads to sizes of $7.5 \mathrm{~nm}$. This trend is also observed for the reflex at $10.6^{\circ}$, which is labeled (100) and is defined by an inplane distance of $8.33 \AA$ A. It shows a slight shift toward larger angles with an increasing amount of TAP in the synthesis. If melamine is completely substituted, this reflex cannot be observed anymore, which has also been reported for carbon nitrides which are prepared by the thermal condensation of TAP or barbituric acid. ${ }^{22}$ The fwhm of the (100) reflex and the

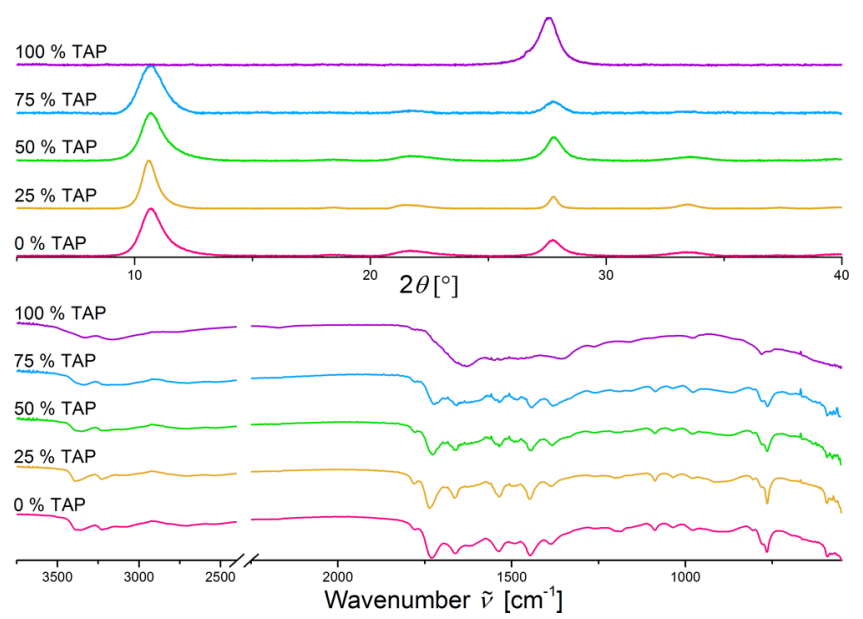

Figure 2. XRD pattern of PTI shows two characteristic reflexes at $10.6^{\circ}$ and $27.7^{\circ}$, which are related to an in-plane periodicity (100) and interlayer stacking (002), respectively (top). FTIR spectra of TAP-doped PTI. The vibration at $2350 \mathrm{~cm}^{-1}$ which is contributed by $\mathrm{CO}_{2}$ has been removed to improve the clarity (bottom).

calculated sizes decrease with increasing molecular doping from $10.5 \mathrm{~nm}$ (0\% TAP) to $6.7 \mathrm{~nm}$ (75\% TAP). All in all in $\mathrm{XRD}$, a decrease in the crystalline quality of the product can be observed in terms of the crystal size in the $a b$-plane as well as along the $c$-axis.

Figure 2 also shows the Fourier transform infrared (FTIR) spectra of the products. The characteristic absorptions of carbon nitrides can be observed. The peak at $805 \mathrm{~cm}^{-1}$ is defined by the out-of-plane bending mode of the triazine ring. The region between 1800 and $1100 \mathrm{~cm}^{-1}$ shows various absorption maxima for imide- and nitride-stretching vibrations. At 1445 and $1260 \mathrm{~cm}^{-1}$, the characteristic bands for $\mathrm{C}=\mathrm{N}$ and $\mathrm{C}-\mathrm{NH}-\mathrm{C}$ vibrations are found, respectively. ${ }^{9}$ The presence of oxygen-containing functional groups such as $\mathrm{C}=\mathrm{O}$ or $\mathrm{C}-\mathrm{OH}$ is indicated by the bands around 1730 and $1180 \mathrm{~cm}^{-1}$ and is in correspondence with the results of the elemental analysis. The incorporation of oxygen has been reported in a previous work and has its origin in the synthesis approach. ${ }^{36}$ The bands at 3350 and $3220 \mathrm{~cm}^{-1}$ are related to NH-stretching vibrations. The peak at $2350 \mathrm{~cm}^{-1}$ can be assigned to carbon dioxide. The FTIR spectra of the $0,25,50$, and $75 \%$ samples are similar apart from an increased broadening of the peaks that can be observed with an increasing TAP amount. This broadening indicates a lower structural order. The similarity of the spectra of TAP-doped carbon nitrides was also recognized by Ho et al. ${ }^{38}$ In contrast, the bands observed for PTI 1660, 1535, 1445, and $1380 \mathrm{~cm}^{-1}$ disappear in the $100 \%$ sample and two broad bands at 1633 and $1355 \mathrm{~cm}^{-1}$ appear. The peak at $1260 \mathrm{~cm}^{-1}$, which is assigned to the $\mathrm{C}-\mathrm{NH}-\mathrm{C}$ vibration, can be observed at low intensities for all of the samples. ${ }^{9}$ The low intensity of this signal combined with the sizes calculated from the XRD pattern shows the low degree of polymerization of the product. The intensity of the out-of-plane bending mode of PTI, which is found at $805 \mathrm{~cm}^{-1}$, decreases with increasing amount of TAP used in the synthesis and cannot be observed for the $100 \%$ sample.

The incorporation of TAP was further verified by elemental analysis. The amounts of carbon, nitrogen, and hydrogen were determined by combustion analysis, whereas the amount of oxygen was provided by pyrolysis analysis. The calculated sum formula of PTI is $\mathrm{C}_{3} \mathrm{~N}_{4.5} \mathrm{H}_{1.5}$. The values found for the samples 
Table 1. Amounts of Carbon, Nitrogen, Hydrogen and Oxygen Found in the Sample by Combustion and Pyrolysis Analysis

$\begin{array}{lccccc} & \mathrm{C} \text { mass \% (atomic \%) } & \mathrm{N} \text { mass \% (atomic \%) } & \mathrm{H} \text { mass \% (atomic \%) } & \text { O mass \% (atomic \%) } & \text { sum formula (integer carbon) } \\ \text { 0\% TAP } & 26.39(20.99) & 48.72(33.23) & 3.61(34.22) & 19.37(11.57) \\ 25 \% \text { TAP } & 27.74(21.79) & 47.57(32.04) & 3.74(35.00) & 18.95(11.17) \\ 50 \% \text { TAP } & 28.43(22.37) & 46.42(31.31) & 3.73(34.97) & 19.21(11.35) \\ 75 \% \text { TAP } & 28.52(22.63) & 45.10(30.69) & 3.70(34.99) & \mathrm{C}_{3} \mathrm{~N}_{4.75} \mathrm{O}_{1.65} \mathrm{H}_{4.89} & 19.61(11.68) \\ 100 \% \text { TAP } & 30.94(24.70) & 43.50(29.78) & 3.53(33.59) & \mathrm{C}_{3} \mathrm{~N}_{4.20} \mathrm{O}_{1.52} \mathrm{H}_{4.69} & \mathrm{C}_{3} \mathrm{~N}_{4.07} \mathrm{O}_{1.55} \mathrm{H}_{4.63} \\ \end{array}$
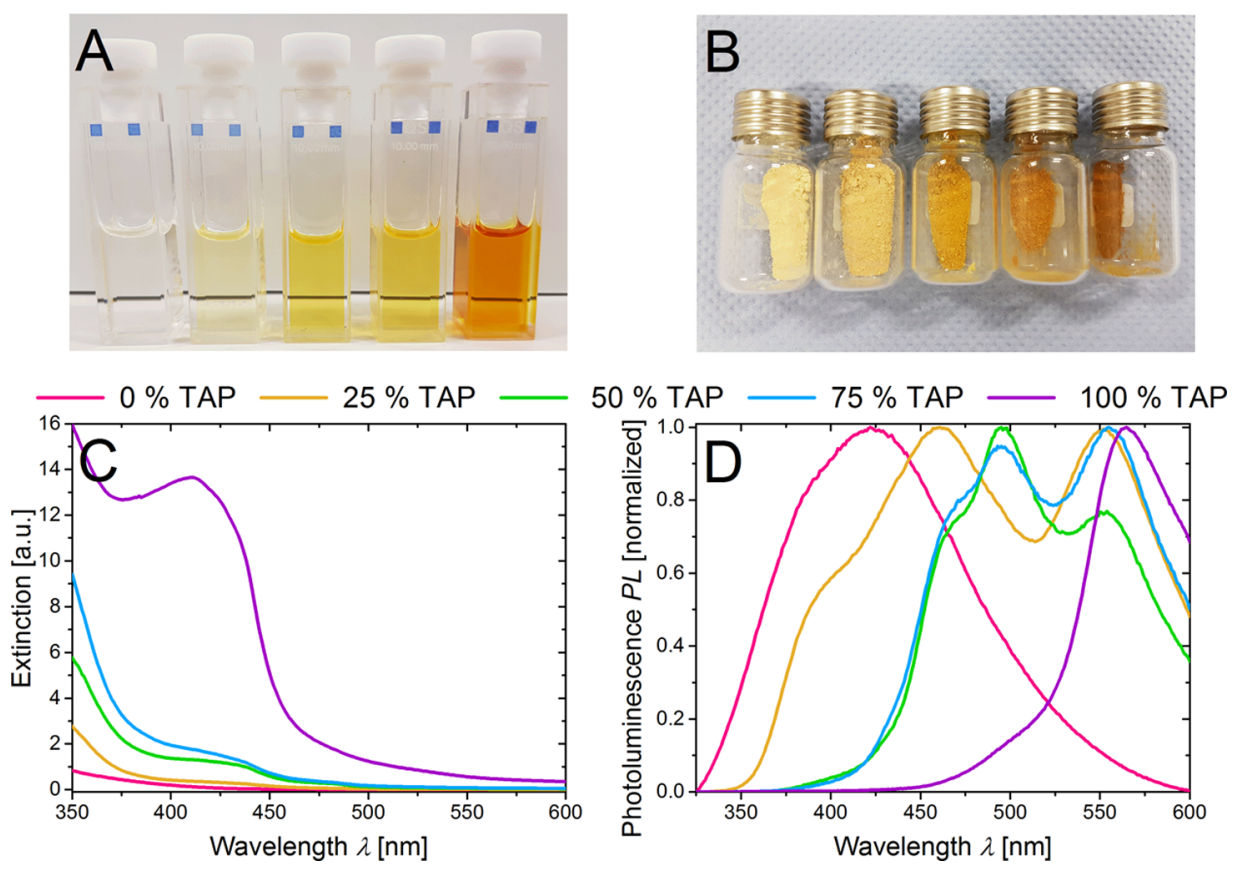

Figure 3. Photographs of the $0,25,50,75$, and $100 \%$ samples (from left to right) in DMSO (A) and freeze-dried (B). UV-vis spectra (C) and PL spectra (D) of the samples measured in DMSO.

with different molar percentages of TAP in the synthesis are presented in Table 1 . The sum formula calculated for $100 \%$ of TAP in the synthesis is $\mathrm{C}_{4} \mathrm{~N}_{3.5} \mathrm{H}_{2.5}$. Oxygen and the increased amount of hydrogen, which are present at a constant value in all the samples, are explained by adsorbed water as well as by the incorporation of oxygen-containing functional groups such as $\mathrm{C}-\mathrm{OH}$ and $\mathrm{COOH}$ because of the synthesis method. ${ }^{36}$ The elemental analysis confirms the low degree of condensation of the as-formed product. The difference from the calculated and the measured nitrogen amount in the doped samples can be explained by terminating amine groups. In general, it can be shown that the $\mathrm{C} / \mathrm{N}$-ratio increases with the addition of TAP.

In ${ }^{13} \mathrm{C}$ NMR spectra, which were recorded in deuterized dimethyl sulfoxide (DMSO), two peaks at 166 and $150 \mathrm{ppm}$ are observed. These peaks can be assigned to the carbon next to the terminal $\mathrm{NH}_{2}$ group and the carbon next to the bridging $\mathrm{NH}$ group. For the 0,25 , and 50\% TAP samples, only these two peaks are observed. The 75\% TAP samples show additional peaks in the region between 166 and $150 \mathrm{ppm}$ that are related to the changes in the chemical environment by the carbon-substituted nitrogen in the triazine ring. This additional carbon atom contributes to the spectrum by the peak at 73 ppm. ${ }^{39}$ For the $100 \%$ TAP samples, only this peak at $73 \mathrm{ppm}$ and the peak at $150 \mathrm{ppm}$ can be observed. The ${ }^{13} \mathrm{C}$ NMR spectra are shown in Figure SI3.

Figure 3A,B shows two photographs of the different samples in liquid and solid state as well as the spectra obtained by UVvis spectroscopy and photoluminescence (PL) measurements in solution (DMSO as the solvent). The photographs show a colorless (solution) or pale yellowish color (solid) for the $0 \%$ sample. With an increasing amount of TAP in the synthesis, the samples turn yellow and orange. The $100 \%$ sample appears brown. These observations are confirmed by the obtained spectra. The absorption spectra are defined by a shoulder at $410 \mathrm{~nm}$, which becomes more pronounced with an increasing amount of TAP used in the synthesis. A shift of the absorbance in the visible range is clearly observable (Figure 3C). The solid-state spectra and the corresponding Tauc plots show a main feature with a Tauc gap between 3.3 and $3.4 \mathrm{eV}$ for the 0 , 25,50 , and $75 \%$ sample. For the $100 \%$ sample, this feature can be found at $3.15 \mathrm{eV}$. A second feature appears at a lower energy of $2.7 \mathrm{eV}$ and can be observed for the 25, 50, 75, and $100 \%$ sample (Figure SI4). Thus, with an increasing amount of $\mathrm{TAP}$, an additional absorption process takes place at lower energies compared to the regular absorption of PTI. The normalized PL spectra show a broad peak at $425 \mathrm{~nm}$ for the product with $0 \%$ of TAP in the synthesis (Figure 3D). This wavelength is slightly red-shifted compared to values reported in the literature. ${ }^{40}$ The complete substitution of melamine by TAP results in a red shift of this peak to $564 \mathrm{~nm}$. The PL spectra for the 25,50 , and $75 \%$ samples consist of several peaks, whose positions can be reconstructed by multiplying the PL spectra of the 0 and $100 \%$ samples. The red shift of the PL is in good agreement with the energy observed for the shoulder in the absorption spectra and the calculated Tauc gap. 

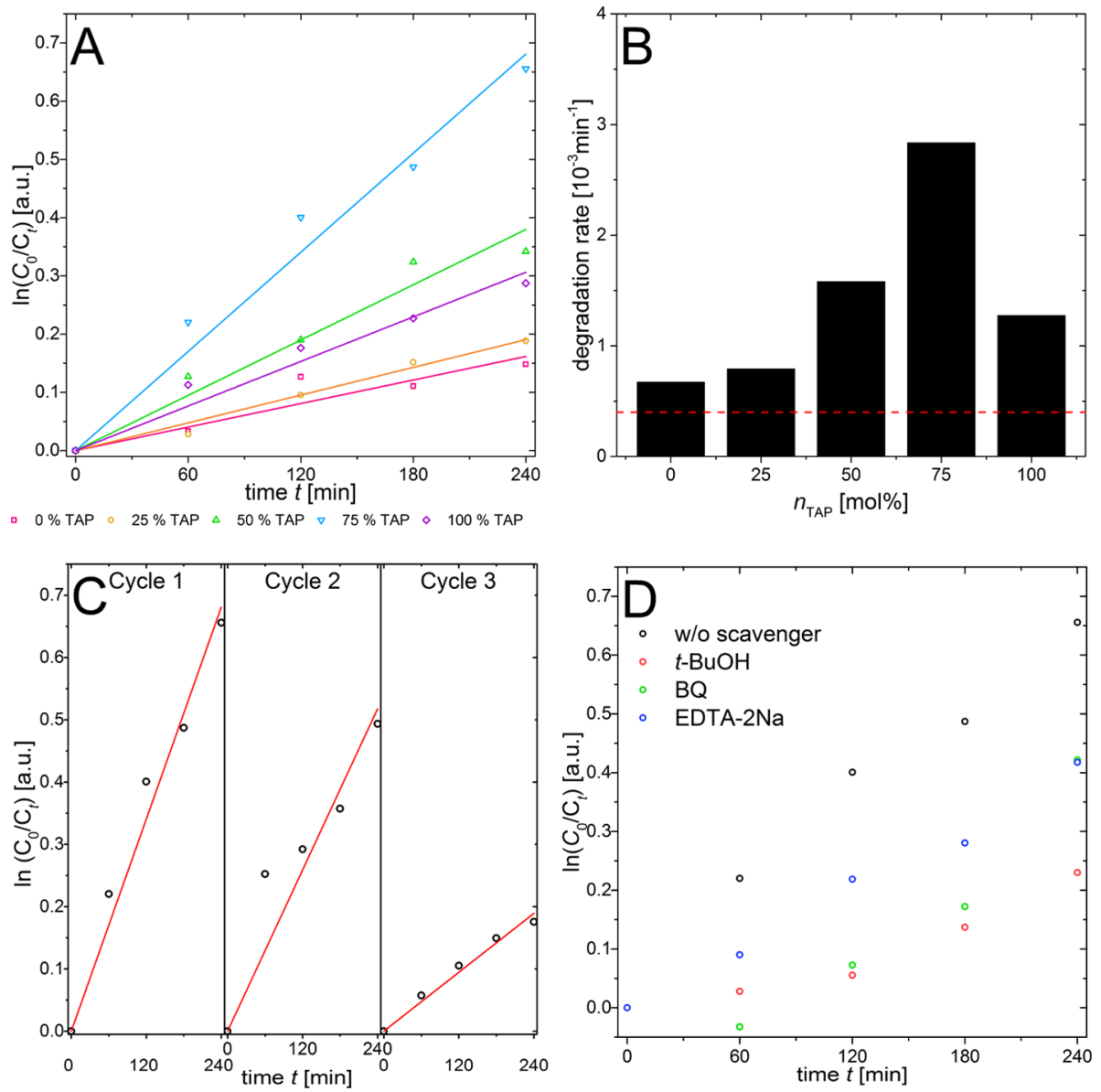

Figure 4. Logarithmic plot of the degradation of $M B$ against time (A) and a comparison of the degradation rates of $M B$ using catalysts with different TAP amounts in the synthesis and melon (dashed red line) (B). Recycling experiments of the 75\% TAP sample for three cycles (C) and scavenger experiments using $t$ - $\mathrm{BuOH}, \mathrm{BQ}$ and EDTA-2Na (D).

Scanning electron microscopy (SEM) and transmission electron microscopy (TEM) images, which are presented in Figure SI5, show no change in the morphology of the carbon nitride. The samples consist of loosely arranged networks made of less than $100 \mathrm{~nm}$ wide ribbon-like structures.

Prior to photocatalytic experiments, the specific surface of the catalyst was determined by Brunauer-Emmett-Teller (BET)-measurements. For the 0, 25, 50, 75, and 100\% TAP samples, specific surfaces of $51.4,45.5,42.5,52.9$, and $53.9 \mathrm{~m}^{2} /$ $\mathrm{g}$ were found, respectively. The observed values are similar and do not show any trend which could be related to the amount of TAP used in the synthesis. Hence, changes in the photocatalytic properties of the different samples cannot be related to the specific surface.

Photocatalytic Experiments. Degradation of MB. All the prepared samples were capable of decomposing $\mathrm{MB}$ without the addition of any cocatalyst. The adsorption/ desorption equilibrium was set after $140 \mathrm{~min}$ stirring in the dark (Figure SI6). The calculated adsorption capacities of the molar amount of $\mathrm{MB}$ per mass of the catalyst are similar for all the samples $(0.051 \pm 0.006 \mathrm{mmol} / \mathrm{g})$ and are in good agreement with the PTI synthesized without sodium hydroxide $(0.04 \mathrm{mmol} / \mathrm{g}) .^{36}$ The multiplication of the adsorption capacity by the Avogadro constant and the size of a $\mathrm{MB}$ molecule of $135 \AA^{2}$ leads to an estimated surface value of 41.5 $\mathrm{m}^{2} / \mathrm{g}$. ${ }^{41}$ This value is close to the specific surfaces found by BET measurements. Therefore, it is assumed that most of the surface is accessible by the $\mathrm{MB}$ molecules and a nearly complete coverage is achieved. The obtained spectra are shown in Figure SI7. The logarithmic plots of the intensities at the absorption maximum of $\mathrm{MB}$ are shown in Figure 4A. The evaluation of the observed degradation rates, which is depicted in Figure 4B, shows an increase depending on the amount of TAP used in the synthesis. The highest rate of $2.8 \times 10^{-3}$ $\mathrm{min}^{-1}$ was observed for the sample, in which $75 \%$ of melamine was substituted by TAP. We were able to boost the degradation rate by a factor of 4 in relation to the rate of $0.6 \times 10^{-3} \mathrm{~min}^{-1}$ of the pure PTI sample. We explain the increased degradation rate by the enhanced overlap of the lamp spectrum and the absorption spectra of the different PTI samples (Figure SI8) as well as the different electronic properties, resulting from the incorporation of TAP. In detail, Bhunia et al. performed density functional theory calculations and showed that TAP doping and the resulting incorporation of carbon result in a smaller band gap by lowering the absolute potential of the valence band. ${ }^{23}$ Nevertheless, the incorporation of melamine in a certain amount seems to be important as the sample prepared by $100 \%$ of TAP shows a lower degradation rate. For comparison, melon was prepared by thermally induced polycondensation of melamine by standard procedures. ${ }^{42}$ The evaluated rate of the degradation of $\mathrm{MB}$ using melamine-derived melon is $0.4 \times 10^{-3} \mathrm{~min}^{-1}$. It is shown that PTI exhibits a slightly higher degradation rate compared to melon and by molecular doping an increase of the rate by a 
factor of 7 is achieved. The stability of the catalyst was evaluated for the $75 \%$ TAP sample by the separation of the catalyst and the $\mathrm{MB}$ solution after every circle, subsequent washing with water, and redispersing of the catalyst in a fresh $\mathrm{MB}$ solution. It was shown that the degradation rate decreases with every recycling step (Figure 4C). After 3 cycles the degradation rate decreases to $27.5 \%$ of the initial value. This decrease cannot be explained by the loss of catalyst material during the washing procedure and may result from adsorbed degradation products that block the active sites of the catalyst. Additional washing steps might improve the recyclability. In addition, scavenger experiments were conducted to identify the active species in the degradation process (Figure 4D). tertButanol $(t-\mathrm{BuOH})$, para-benzoquinone (BQ), and EDTA$2 \mathrm{Na}$ have been added as scavengers for hydroxyl radicals, superoxide radicals, and holes, respectively. ${ }^{43,44}$ The addition of $t$ - $\mathrm{BuOH}$ results in a slightly decreased degradation rate, whereas for $\mathrm{BQ}$ for the first hour no degradation of $\mathrm{MB}$ but a shift in the absorption of BQ is observed (Figure SI7). This shift decreases during the $4 \mathrm{~h}$ of degradation and the rate of the degradation of $\mathrm{MB}$ increases. We assume that evolving superoxide radicals react preferentially with $\mathrm{BQ}$. When most of the $\mathrm{BQ}$ molecules have reacted, the $\mathrm{MB}$ degradation starts to take place. The observed rate is similar to the reference without any scavenger molecule. These results are in accordance to our previous work. ${ }^{36}$ The addition of EDTA$2 \mathrm{Na}$ effects a small decrease of the degradation rate. Thus, it is concluded that superoxide radicals are the main active species of the degradation of $\mathrm{MB}$ followed by hydroxyl radicals and holes.

\section{CONCLUSIONS}

In summary, we report a facile modification of the electrochemical synthesis approach recently reported by the authors, which is based on the formation of radicals during electrolysis of water and the reaction of those with melamine. ${ }^{36}$ Just by partially substituting melamine with TAP in the synthesis, it was possible to further improve the photocatalytic properties of PTI. We were able to tune the molar ratio of melamine and TAP from 0 to $100 \%$. With an increasing amount of TAP, the electronic structure of PTI has been tuned and a decrease of the band gap could be observed. The smaller band gap results in changes in the optical properties of the product in regard to a red-shift in the visible region that can be observed in absorption as well as in PL experiments. This red-shift enhanced the photocatalytic properties of PTI and in dye degradation experiments we were able to observe an increase of the degradation rate by a factor of 4 in relation to the undoped PTI or 7 if compared to melon prepared by thermally induced polycondensation of melamine.

\section{EXPERIMENTAL SECTION}

Chemicals. Melamine (99\%) was purchased from SigmaAldrich, TAP (>97\%) was purchased from Sigma-Aldrich, sodium hydroxide (97\%) was purchased from Grüssing $\mathrm{GmbH}$, hydrochloric acid (37\%) was purchased from VWR, DMSO (99.7\%) was purchased from ACROS organics, MB was purchased from Merck Chemicals $\mathrm{GmbH}$, tert-butanol (99\%) was purchased from Grüssing $\mathrm{GmbH}$, EDTA-2Na (>98.5\%) was purchased from Sigma-Aldrich, and BQ was purchased from Sigma-Aldrich and recrystallized in 2-propanol.
Synthesis of Melon. Melon was synthesized by thermalinduced polycondensation of melamine. $5 \mathrm{~g}(39.6 \mathrm{mmol})$ of melamine was placed in a semiclosed crucible and heated to $550{ }^{\circ} \mathrm{C}$ with a ramp rate of $5{ }^{\circ} \mathrm{C} / \mathrm{min}$. After $2 \mathrm{~h}$ at $550{ }^{\circ} \mathrm{C}$, the product was allowed to cool down naturally, milled into a powder using a mortar and pestle, washed with $100 \mathrm{~mL}$ boiling water, and dried in a vacuum furnace at $60{ }^{\circ} \mathrm{C}$.

Synthesis of PTI. The synthesis is similar to the synthesis for PTI that has been recently reported. ${ }^{36} 630 \mathrm{mg}$ ( $\left.5 \mathrm{mmol}\right)$ of melamine and $800 \mathrm{mg}(20 \mathrm{mmol})$ of sodium hydroxide were dissolved in $100 \mathrm{~mL}$ of demineralized water at room temperature under vigorous stirring. In a three-electrode setup with a reference electrode $(\mathrm{Ag} / \mathrm{AgCl} / \mathrm{KCl})$, counter electrode (platinum), and working electrode (platinum), a voltage of $5 \mathrm{~V}$ was applied for $90 \mathrm{~min}$ at room temperature under continuous stirring. The distance between the counter and working electrode was $0.5 \mathrm{~cm}$. The current was in the range of $600 \mathrm{~mA}$. A photograph of the set-up is shown in Figure SI1.

The product was obtained in solution. By addition of hydrochloric acid, the $\mathrm{pH}$ value was adjusted to obtain a suspension. The product was separated by centrifugation for 5 $\mathrm{min}$ at $7500 \mathrm{rpm}$ (13206 rcf) and washed twice with $30 \mathrm{~mL}$ hot demineralized water.

TAP was used for molecular doping. The synthesis was as described above, but melamine was substituted by $0,25,50,75$ and $100 \mathrm{~mol} \%$ of TAP. Additionally, the duration of the reaction was changed to $120,210,240,330$ and $300 \mathrm{~min}$, respectively.

Photocatalytic Experiments. $50 \mathrm{mg}$ of the different catalyst materials was dispersed in $100 \mathrm{~mL}$ of a $10 \mathrm{mg} / \mathrm{L} \mathrm{MB}$ in water solution and stirred for $140 \mathrm{~min}$ in the dark to set an adsorption/desorption equilibrium. As a light source, a $300 \mathrm{~W}$ xenon lamp (UXL-302-O) combined with a water filter to avoid heating effects by infrared radiation was used. Aliquots of $4 \mathrm{~mL}$ were taken every $60 \mathrm{~min}$. The aliquots were centrifuged once at $11000 \mathrm{rpm}$ (28408 rcf) in order to remove the catalyst material. The supernatant was filtered using a hydrophilic syringe filter (nylon, $0.2 \mu \mathrm{m}$ pore size).

For recycling experiments, the catalyst was collected after every cycle and separated from the $M B$ solution by centrifugation at $11000 \mathrm{rpm}(28408 \mathrm{rcf})$ for $15 \mathrm{~min}$ at a temperature of $5{ }^{\circ} \mathrm{C}$. The supernatant was discarded. The catalyst was redispersed in $30 \mathrm{~mL}$ of water and after the adsorption/desorption equilibrium, it was set again separated from the solution by centrifugation. This procedure was repeated twice. The second and third circle of degradation was conducted as described above.

The reactive species in the degradation process were identified by adding tert-butanol (10 vol \%), EDTA-2Na (1 $\mathrm{mmol})$ or BQ $(1 \mathrm{mmol})$ to the experiment.

Methods. UV-Vis-Spectroscopy. A quartz cuvette with an optical path length of $10 \mathrm{~mm}$ was used for absorption and PL experiments. UV-vis absorption spectra were obtained with a PerkinElmer LAMBDA 25 spectrometer in a two-beam set-up. PL spectra were obtained with a HORIBA FluoroMax-4 spectrometer.

Fourier Transform Infrared Spectroscopy. The FTIR spectra were collected on a Varian 660 infrared spectrometer with an attenuated total reflection sampling technique. The set-up was purged with nitrogen for $1 \mathrm{~h} .32$ scans were used to collect the spectrum. 
X-ray Diffraction. Powder XRD measurements were carried out on a Philips X'Perts PRO MPD diffractometer with monochromatic X-ray radiation from a copper anode with a wavelength of $1.54 \AA(\mathrm{Cu} \mathrm{K} \alpha)$. The samples were dispersed in water, drop casted. and dried on a $\langle 911\rangle$ or $\langle 711\rangle$ silicon wafer.

Electronmicroscopy. SEM images were collected with a Zeiss MA10 electron microscope with $\mathrm{a} \mathrm{LaB}_{6}$ emitter at $5 \mathrm{kV}$. TEM images were collected on JEOL-1011 with a thermal emitter operated at an acceleration voltage of $100 \mathrm{kV}$.

Gas Adsorption. The BET surface area was obtained by nitrogen sorption experiments using a Quantachrome Autosorb iQ Automated Gas Sorption System at $77 \mathrm{~K}$.

NMR Spectroscopy. ${ }^{13} \mathrm{C}$ NMR spectra were recorded in DMSO in a ${ }^{13} \mathrm{C}\left\{{ }^{1} \mathrm{H}\right\}$-BB experiment using 3072 scans on a Bruker AVANCE $400 \mathrm{MHz}$ spectrometer.

\section{ASSOCIATED CONTENT}

\section{S Supporting Information}

The Supporting Information is available free of charge on the ACS Publications website at DOI: 10.1021/acsomega.8b02659.

Photograph of the experimental set-up, additional UVvis spectra and Tauc plots, ${ }^{13} \mathrm{C}$ NMR spectra, SEM and TEM images, UV-vis spectra of aliquots taken during dye degradation, and xenon lamp spectrum (PDF)

\section{AUTHOR INFORMATION}

\section{Corresponding Author}

*E-mail: christian.klinke@swansea.ac.uk.

\section{ORCID}

Leonard Heymann: 0000-0001-6177-6943

Christian Klinke: 0000-0001-8558-7389

\section{Notes}

The authors declare no competing financial interest.

\section{ACKNOWLEDGMENTS}

L.H. and C.K. gratefully acknowledge financial support of the European Research Council via the ERC Starting Grant "2DSYNETRA" (Seventh Framework Program FP7, Project: 304980). C.K. thanks the German Research Foundation DFG for financial support in the frame of the Cluster of Excellence "Center of ultrafast imaging CUI" and the Heisenberg scholarship KL 1453/9-2.

\section{REFERENCES}

(1) Wang, X.; Maeda, K.; Thomas, A.; Takanabe, K.; Xin, G.; Carlsson, J. M.; Domen, K.; Antonietti, M. A Metal-Free Polymeric Photocatalyst for Hydrogen Production from Water under Visible Light. Nat. Mater. 2009, 8, 76-80.

(2) Lotsch, B. V.; Döblinger, M.; Sehnert, J.; Seyfarth, L.; Senker, J.; Oeckler, O.; Schnick, W. Unmasking Melon by a Complementary Approach Employing Electron Diffraction, Solid-State NMR Spectroscopy, and Theoretical Calculations - Structural Characterization of a Carbon Nitride Polymer. Chem.-Eur. J. 2007, 13, 4969-4980.

(3) Bojdys, M. J.; Müller, J.-O.; Antonietti, M.; Thomas, A. Ionothermal Synthesis of Crystalline, Condensed, Graphitic Carbon Nitride. Chem.-Eur. J. 2008, 14, 8177-8182.

(4) Yan, S. C.; Li, Z. S.; Zou, Z. G. Photodegradation Performance of g- $\mathrm{C}_{3} \mathrm{~N}_{4}$ Fabricated by Directly Heating Melamine. Langmuir 2009, 25, 10397-10401.

(5) Liu, J.; Zhang, T.; Wang, Z.; Dawson, G.; Chen, W. Simple Pyrolysis of Urea into Graphitic Carbon Nitride with Recyclable
Adsorption and Photocatalytic Activity. J. Mater. Chem. 2011, 21, $14398-14401$.

(6) Wirnhier, E.; Döblinger, M.; Gunzelmann, D.; Senker, J.; Lotsch, B. V.; Schnick, W. Poly(Triazine Imide) with Intercalation of Lithium and Chloride Ions $\left[\left(\mathrm{C}_{3} \mathrm{~N}_{3}\right)_{2}\left(\mathrm{NH}_{\mathrm{x}} \mathrm{Li}_{1-\mathrm{x}}\right)_{3} \cdot \mathrm{LiCl}\right]$ : A Crystalline 2D Carbon Nitride Network. Chem.-Eur. J. 2011, 17, 3213-3221.

(7) Ham, Y.; Maeda, K.; Cha, D.; Takanabe, K.; Domen, K. Synthesis and Photocatalytic Activity of Poly(Triazine Imide). Chem.-Asian J. 2012, 8, 218-224.

(8) Schwinghammer, K.; Tuffy, B.; Mesch, M. B.; Wirnhier, E.; Martineau, C.; Taulelle, F.; Schnick, W.; Senker, J.; Lotsch, B. V. Triazine-Based Carbon Nitrides for Visible-Light-Driven Hydrogen Evolution. Angew. Chem., Int. Ed. 2013, 52, 2435-2439.

(9) Schwinghammer, K.; Mesch, M. B.; Duppel, V.; Ziegler, C.; Senker, J.; Lotsch, B. V. Crystalline Carbon Nitride Nanosheets for Improved Visible-Light Hydrogen Evolution. J. Am. Chem. Soc. 2014, 136, 1730-1733.

(10) Savateev, A.; Pronkin, S.; Epping, J. D.; Willinger, M. G.; Wolff, C.; Neher, D.; Antonietti, M.; Dontsova, D. Potassium Poly(Heptazine Imides) from Aminotetrazoles: Shifting Band Gaps of Carbon Nitride-like Materials for More Efficient Solar Hydrogen and Oxygen Evolution. ChemCatChem 2016, 9, 167-174.

(11) Döblinger, M.; Lotsch, B. V.; Wack, J.; Thun, J.; Senker, J.; Schnick, W. Structure Elucidation of Polyheptazine Imide by Electron Diffraction-a Templated 2D Carbon Nitride Network. Chem. Commun. 2009, 1541.

(12) Chen, Z.; Savateev, A.; Pronkin, S.; Papaefthimiou, V.; Wolff, C.; Willinger, M. G.; Willinger, E.; Neher, D.; Antonietti, M.; Dontsova, D. "The Easier the Better" Preparation of Efficient Photocatalysts-Metastable Poly(Heptazine Imide) Salts. Adv. Mater. 2017, 29, 1700555.

(13) Algara-Siller, G.; Severin, N.; Chong, S. Y.; Björkman, T.; Palgrave, R. G.; Laybourn, A.; Antonietti, M.; Khimyak, Y. Z.; Krasheninnikov, A. V.; Rabe, J. P.; Kaiser, U.; Cooper, A. I.; Thomas, A.; Bojdys, M. J. Triazine-Based Graphitic Carbon Nitride: A TwoDimensional Semiconductor. Angew. Chem., Int. Ed. 2014, 53, 74507455.

(14) Kessler, F. K.; Zheng, Y.; Schwarz, D.; Merschjann, C.; Schnick, W.; Wang, X.; Bojdys, M. J. Functional Carbon Nitride MaterialsDesign Strategies for Electrochemical Devices. Nat. Rev. Mater. 2017, 2, 17030 .

(15) Kumar, P.; Boukherroub, R.; Shankar, K. Sunlight-Driven Water-Splitting Using Two-Dimensional Carbon Based Semiconductors. J. Mater. Chem. A 2018, 6, 12876-12931.

(16) Sun, L.; Yang, M.; Huang, J.; Yu, D.; Hong, W.; Chen, X. Freestanding Graphitic Carbon Nitride Photonic Crystals for Enhanced Photocatalysis. Adv. Funct. Mater. 2016, 26, 4943-4950.

(17) Yan, H. Soft-Templating Synthesis of Mesoporous Graphitic Carbon Nitride with Enhanced Photocatalytic $\mathrm{H}_{2}$ Evolution under Visible Light. Chem. Commun. 2012, 48, 3430.

(18) Yang, Z.; Zhang, Y.; Schnepp, Z. Soft and Hard Templating of Graphitic Carbon Nitride. J. Mater. Chem. A 2015, 3, 14081-14092.

(19) Zhang, J.; Chen, X.; Takanabe, K.; Maeda, K.; Domen, K.; Epping, J. D.; Fu, X.; Antonietti, M.; Wang, X. Synthesis of a Carbon Nitride Structure for Visible-Light Catalysis by Copolymerization. Angew. Chem., Int. Ed. 2009, 49, 441-444.

(20) Xing, W.; Chen, G.; Li, C.; Han, Z.; Hu, Y.; Meng, Q. Doping Effect of Non-Metal Group in Porous Ultrathin g- $\mathrm{C}_{3} \mathrm{~N}_{4}$ Nanosheets towards Synergistically Improved Photocatalytic Hydrogen Evolution. Nanoscale 2018, 10, 5239-5245.

(21) Bhunia, M. K.; Yamauchi, K.; Takanabe, K. Harvesting Solar Light with Crystalline Carbon Nitrides for Efficient Photocatalytic Hydrogen Evolution. Angew. Chem., Int. Ed. 2014, 53, 11001-11005.

(22) Vorobyeva, E.; Chen, Z.; Mitchell, S.; Leary, R. K.; Midgley, P.; Thomas, J. M.; Hauert, R.; Fako, E.; López, N.; Pérez-Ramírez, J. Tailoring the Framework Composition of Carbon Nitride to Improve the Catalytic Efficiency of the Stabilised Palladium Atoms. J. Mater. Chem. A 2017, 5, 16393-16403. 
(23) Bhunia, M. K.; Melissen, S.; Parida, M. R.; Sarawade, P.; Basset, J.-M.; Anjum, D. H.; Mohammed, O. F.; Sautet, P.; Le Bahers, T.; Takanabe, K. Dendritic Tip-on Polytriazine-Based Carbon Nitride Photocatalyst with High Hydrogen Evolution Activity. Chem. Mater. 2015, 27, 8237-8247.

(24) Zhang, J.; Zhang, G.; Chen, X.; Lin, S.; Möhlmann, L.; Dołęga, G.; Lipner, G.; Antonietti, M.; Blechert, S.; Wang, X. Co-Monomer Control of Carbon Nitride Semiconductors to Optimize Hydrogen Evolution with Visible Light. Angew. Chem., Int. Ed. 2012, 51, 31833187.

(25) Guo, S.; Zhu, Y.; Yan, Y.; Min, Y.; Fan, J.; Xu, Q. Holey Structured Graphitic Carbon Nitride Thin Sheets with Edge Oxygen Doping via Photo-Fenton Reaction with Enhanced Photocatalytic Activity. Appl. Catal., B 2016, 185, 315-321.

(26) Zhang, P.; Li, X.; Shao, C.; Liu, Y. Hydrothermal Synthesis of Carbon-Rich Graphitic Carbon Nitride Nanosheets for Photoredox Catalysis. J. Mater. Chem. A 2015, 3, 3281-3284.

(27) Lan, D.-H.; Wang, H.-T.; Chen, L.; Au, C.-T.; Yin, S.-F. Phosphorous-Modified Bulk Graphitic Carbon Nitride: Facile Preparation and Application as an Acid-Base Bifunctional and Efficient Catalyst for $\mathrm{CO}_{2}$ cycloaddition with Epoxides. Carbon 2016, 100, 81-89.

(28) Xu, C.; Han, Q.; Zhao, Y.; Wang, L.; Li, Y.; Qu, L. SulfurDoped Graphitic Carbon Nitride Decorated with Graphene Quantum Dots for an Efficient Metal-Free Electrocatalyst. J. Mater. Chem. A 2015, 3, 1841-1846.

(29) Raziq, F.; Qu, Y.; Zhang, X.; Humayun, M.; Wu, J.; Zada, A.; Yu, H.; Sun, X.; Jing, L. Enhanced Cocatalyst-Free Visible-Light Activities for Photocatalytic Fuel Production of $\mathrm{g}-\mathrm{C}_{3} \mathrm{~N}_{4}$ by Trapping Holes and Transferring Electrons. J. Phys. Chem. C 2015, 120, 98107.

(30) Lin, Z.; Wang, X. Nanostructure Engineering and Doping of Conjugated Carbon Nitride Semiconductors for Hydrogen Photosynthesis. Angew. Chem., Int. Ed. 2013, 52, 1735-1738.

(31) Han, Q.; Hu, C.; Zhao, F.; Zhang, Z.; Chen, N.; Qu, L. OneStep Preparation of Iodine-Doped Graphitic Carbon Nitride Nanosheets as Efficient Photocatalysts for Visible Light Water Splitting. J. Mater. Chem. A 2015, 3, 4612-4619.

(32) Zhang, G.; Zhang, M.; Ye, X.; Qiu, X.; Lin, S.; Wang, X. Iodine Modified Carbon Nitride Semiconductors as Visible Light Photocatalysts for Hydrogen Evolution. Adv. Mater. 2013, 26, 805-809.

(33) Wang, Y.; Di, Y.; Antonietti, M.; Li, H.; Chen, X.; Wang, X. Excellent Visible-Light Photocatalysis of Fluorinated Polymeric Carbon Nitride Solids. Chem. Mater. 2010, 22, 5119-5121.

(34) Ong, W.-J.; Tan, L.-L.; Ng, Y. H.; Yong, S.-T.; Chai, S.-P. Graphitic Carbon Nitride $\left(\mathrm{g}-\mathrm{C}_{3} \mathrm{~N}_{4}\right)$-Based Photocatalysts for Artificial Photosynthesis and Environmental Remediation: Are We a Step Closer to Achieving Sustainability? Chem. Rev. 2016, 116, 71597329.

(35) Wang, X.; Chen, X.; Thomas, A.; Fu, X.; Antonietti, M. MetalContaining Carbon Nitride Compounds: A New Functional OrganicMetal Hybrid Material. Adv. Mater. 2009, 21, 1609-1612.

(36) Heymann, L.; Schiller, B.; Noei, H.; Stierle, A.; Klinke, C. A New Synthesis Approach for Carbon Nitrides: Poly(Triazine Imide) and Its Photocatalytic Properties. ACS Omega 2018, 3, 3892-3900.

(37) Lu, Q.; Deng, J.; Hou, Y.; Wang, H.; Li, H.; Zhang, Y. One-Step Electrochemical Synthesis of Ultrathin Graphitic Carbon Nitride Nanosheets and Its Application to the Detection of Uric Acid. Chem. Commun. 2015, 51, 12251-12253.

(38) Ho, W.; Zhang, Z.; Lin, W.; Huang, S.; Zhang, X.; Wang, X.; Huang, Y. Copolymerization with 2,4,6-Triaminopyrimidine for the Rolling-up the Layer Structure, Tunable Electronic Properties, and Photocatalysis of $\mathrm{g}-\mathrm{C}_{3} \mathrm{~N}_{4}$. ACS Appl. Mater. Interfaces 2015, 7, 54975505.

(39) Németh, B.; Wéber, C.; Veszprémi, T.; Gáti, T.; Demeter, Á. Carbon Protonation of 2,4,6-Triaminopyrimidines: Synthesis, NMR Studies, and Theoretical Calculations. J. Org. Chem. 2006, 71, 49104918.
(40) Miller, T. S.; Suter, T. M.; Telford, A. M.; Picco, L.; Payton, O. D.; Russell-Pavier, F.; Cullen, P. L.; Sella, A.; Shaffer, M. S. P.; Nelson, J.; Tileli, V.; McMillan, P. F.; Howard, C. A. Single Crystal, Luminescent Carbon Nitride Nanosheets Formed by Spontaneous Dissolution. Nano Lett. 2017, 17, 5891-5896.

(41) Arias, M.; López, E.; Nuñez, A.; Rubinos, D.; Soto, B.; Barral, M. T.; Díaz-Fierros, F. Adsorption of Methylene Blue by Red Mud, An Oxide- Rich Byproduct of Bauxite Refining. Effect of MineralOrganic-Microorganism Interactions on Soil and Freshwater Environments; Springer US: Boston, MA, 1999; pp 361-365.

(42) Ou, H.; Lin, L.; Zheng, Y.; Yang, P.; Fang, Y.; Wang, X. Tri- s -Triazine-Based Crystalline Carbon Nitride Nanosheets for an Improved Hydrogen Evolution. Adv. Mater. 2017, 29, 1700008.

(43) Li, Y.; Jin, R.; Li, G.; Liu, X.; Yu, M.; Xing, Y.; Shi, Z. Preparation of Phenyl Group Functionalized g- $\mathrm{C}_{3} \mathrm{~N}_{4}$ Nanosheets with Extended Electron Delocalization for Enhanced Visible-Light Photocatalytic Activity. New J. Chem. 2018, 42, 6756-6762.

(44) Bai, X.; Wang, L.; Zong, R.; Zhu, Y. Photocatalytic Activity Enhanced via g- $\mathrm{C}_{3} \mathrm{~N}_{4}$ Nanoplates to Nanorods. J. Phys. Chem. C 2013, 117, 9952-9961. 